\title{
On Thermodynamic and Microscopic Reversibility
}

\begin{abstract}
The word "reversible" has two (apparently) distinct applications in statistical thermodynamics. A thermodynamically reversible process indicates an experimental protocol for which the entropy change is zero, whereas the principle of microscopic reversibility asserts that the probability of any trajectory of a system through phase space equals that of the time reversed trajectory. However, these two terms are actually synonymous: a thermodynamically reversible process is microscopically reversible, and vice versa. $\ddagger$
\end{abstract}

\section{Gavin E. Crooks}

Physical Biosciences Division, Lawrence Berkeley National Laboratory, Berkeley, CA 94720

$\ddagger$ DISCLAIMER :This document was prepared as an account of work sponsored by the United States Government. While this document is believed to contain correct information, neither the United States Government nor any agency thereof, nor the Regents of the University of California, nor any of their employees, makes any warranty, express or implied, or assumes any legal responsibility for the accuracy, completeness, or usefulness of any information, apparatus, product, or process disclosed, or represents that its use would not infringe privately owned rights. Reference herein to any specific commercial product, process, or service by its trade name, trademark, manufacturer, or otherwise, does not necessarily constitute or imply its endorsement, recommendation, or favoring by the United States Government or any agency thereof, or the Regents of the University of California. The views and opinions of authors expressed herein do not necessarily state or reflect those of the United States Government or any agency thereof or the Regents of the University of California. 


\section{Thermodynamic reversibility}

The concept of a thermodynamically reversible process was crucial to the $19^{\text {th }}$ century development of thermodynamics $[1,2]$. A reversible thermodynamic process is one that can proceed equally well either forward or backward, which can occur only if the dissipation, the total entropy change, is zero.

We need to consider a system driven from one thermodynamic equilibrium to another by a changing external perturbation. We can denote the controllable parameters of the system as $\lambda(t)$ for $t \in[a, b]$, and the overall experimental protocol, describing the time course of the control, as $\Lambda$. We assume that the control parameters are unchanging outside the time interval $[a, b]$, so that the system starts at equilibrium at time $a$ and relaxes back to equilibrium at some time $\tau \gg b$. Every protocol also has a conjugate, time-reversed protocol $\tilde{\Lambda}$ where the system begins in thermal equilibrium at $\lambda(b)$ and the control parameters retrace the same series of changes, in reverse, back to $\lambda(a)$.

The second law, the central tenet of thermodynamics, states that the mean total entropy change for any such protocol is non-negative,

$$
\left\langle\Delta S^{\text {total }}\right\rangle_{\Lambda} \geq 0 \text {. }
$$

The total entropy change includes the entropy change of the system and any concomitant entropy change of the environment, for instance due to exchange of energy in the form of heat. The average is taken over many realizations of the experiment. The second law defines the directionality of time $[3,4]$, and appears to be the only physical law that breaks time symmetry in a non-trivial manner. The direction of time we call the future is that in which the entropy increases (on average).

A thermodynamically reversibly process is one with zero net entropy change. Because there is no time directionality in this case, both the forward and reversed protocol can be performed without any dissipation. In practice, this can only be achieved with a quasi-static process, where the active perturbation of the system is spread over a long time span, so that the system is always very close to equilibrium [5].

\section{Microscopic reversibility}

In the early decades of the $20^{\text {th }}$ century, it became apparent, from considerations of chemical kinetics and quantum mechanics, that a type of dynamic, stochastic reversibility must hold on the microscopic level [6-11]. For instance, in 1924 G. N. Lewis stated [8]:

Corresponding to every individual process there is a reverse process, and in a state of equilibrium the average rate of every process is equal to the average rate of its reverse process.

Expressed another way, this Principle of Microscopic Reversibility at Equilibrium $[10,11]$ states that the probability of observing any trajectory at equilibrium is equal to that of observing the time reversed trajectory, under the same conditions,

$$
P[X \mid \lambda]=P[\tilde{X} \mid \lambda] \text {. }
$$

Here, $X$ denotes a trajectory of the system through phase space, $\tilde{X}$ denotes the conjugate, time reversed trajectory, $\lambda$ denotes the fixed external constraints of the system and $P[X \mid \lambda]$ is the probability of observing the given trajectory. 
A related dynamic property is that of Detailed Balance [6, 11, 12], which states that at thermodynamic equilibrium the probability of starting in a state $x$ and transitioning to state $x^{\prime}$ is equal to the reversed transition.

$$
P(x \mid \lambda) P\left(x^{\prime} \mid x, \lambda\right)=P\left(x^{\prime} \mid \lambda\right) P\left(x \mid x^{\prime}, \lambda\right)
$$

Detailed balance is a statement about the flow of probability between states, whereas microscopic reversibility is a statement about the relative probability of trajectories. Howsoever, since any state transition must proceed via a collection of trajectories, and any trajectory is simple a sequence of states, the two principles are essentially equivalent [11], and the terms are often used interchangeable in modern discourse.

\section{Dynamic irreversibility}

We can connect the principles of thermodynamic and microscopic reversibility through the more general principle of dynamic irreversibility [13-17]. In a driven, nonequilibrium process, the probability ratio of a trajectory under the forward protocol, and the reversed trajectory under the conjugate protocol is not unity, but is instead given by the exponential of the total change in entropy along the forward process,

$$
\frac{P[X \mid \Lambda]}{P[\tilde{X} \mid \tilde{\Lambda}]}=e^{\Delta S^{\text {total }}}
$$

We require that the system starts at thermodynamic equilibrium, is connected to an equilibrium thermal environment, and eventually relaxes back to equilibrium after the active perturbation of the system ceases. The total entropy change is a quantitative measure of the breaking of time-reversal symmetry at the level of trajectories.

We can make the connection between thermodynamic and microscopic reversibility by noting that the relative entropy $D(p \| q)=\sum_{i} p_{i} \ln \frac{p_{i}}{q_{i}}$ between the forward and reverse trajectory ensembles is equal to the average total entropy change [18-27]:

$$
\begin{aligned}
D(P[X \mid \Lambda] \| P[\tilde{X} \mid \tilde{\Lambda}]) & =\sum_{X} P[X \mid \Lambda] \ln \frac{P[X \mid \Lambda]}{P[\tilde{X} \mid \tilde{\Lambda}]} \\
& =\sum_{X} P[X \mid \Lambda] \Delta S^{\text {total }} \\
& =\left\langle\Delta S^{\text {total }}\right\rangle_{\Lambda}
\end{aligned}
$$

We can recover the Second Law (1) by noting that relative entropy is non-negative, $D(p ; q) \geq 0$, due to Jensens inequality [28]. Moreover, the relative entropy is equal to zero if, and only if, the two distributions are identical [28]. It follows that the dissipation is zero (and the process is thermodynamically reversible) only if the conjugate forward and reverse trajectories have identical probabilities, and vice versa,

$$
P[X \mid \Lambda]=P[\tilde{X} \mid \tilde{\Lambda}] \Longleftrightarrow\left\langle\Delta S^{\text {total }}\right\rangle=0 \text {. }
$$

Note that essentially we have generalized microscopic reversibility (2) from stationary, equilibrium ensembles to quasi-static processes. Thermodynamic reversibility and microscopic reversibility are equivalent statements about the behavior of a system [17], the first couched in terms of the average behavior of the system, the second in terms of individual trajectories. 


\section{Acknowledgments}

The author thanks John D. Chodera (University of California, Berkeley) and David A. Sivak (Lawrence Berkeley Natl. Lab.) for enlightening discussions and constructive feedback on the manuscript. This work is supported by the Director, Office of Science, Office of Basic Energy Sciences of the U.S. Department of Energy under Contract No. DE-AC02-05CH11231.

\section{References}

[1] Carnot S 1824 Réflexions sur la puissance motrice du feu et sur les machines propres a développer cette puissance (Paris: Chez Bachelier, Libraire) reflections on the motive power of fire and machines fitted to develop that power

[2] Clausius R 1865 Annalen der Physik und Chemie 201 353-400

[3] Eddington A S 1928 The nature of the physical world (Cambridge: Cambridge University Press)

[4] Price H 1996 Time's arrow and Archimedes' point (New York: Oxford University Press)

[5] Callen H B 1985 Thermodynamics and an Introduction to Thermostatistics 2nd ed (New York: Wiley)

[6] Dirac P A M 1924 Proc. Rol. Soc. A. 106 581-596

[7] Tolman R C 1924 Phys. Rev. 23 693-709

[8] Lewis G N 1925 Proc. Natl. Acad. Sci. U.S.A. 11 179-183

[9] Fowler R H and Milne E A 1925 Proc. Natl. Acad. Sci. U.S.A. 11 400-402

[10] Tolman R C 1925 Proc. Natl. Acad. Sci. U.S.A. 11 436-439

[11] Tolman R C 1938 The principles of statistical mechanics (London: Oxford University Press)

[12] Onsager L 1931 Phys. Rev. 37 405-426

[13] Crooks G E 1998 J. Stat. Phys. 90 1481-1487

[14] Crooks G E 1999 Phys. Rev. E 60 2721-2726

[15] Kurchan J 1998 J. Phys. A 31 3719-3729

[16] Lebowitz J L and Spohn H 1999 J. Stat. Phys. 95 333-365

[17] Maes C and Netǒcný K 2003 J. Stat. Phys. 110 269-310

[18] Gaspard P 2004 J. Stat. Phys. 117 599-615

[19] Gaspard P 2004 J. Chem. Phys. 120 8898-8905

[20] Jarzynski C 2006 Phys. Rev. E 73046105

[21] Kawai R, Parrondo J M R and Van den Broeck C 2007 Phys. Rev. Lett. 98080602

[22] Gomez-Marin A, Parrondo J M R and Van den Broeck C 2008 Europhys. Lett. 8250002

[23] Andrieux D, Gaspard P, Ciliberto S, Garnier N, Joubaud S and Petrosyan A 2008 J. Stat. Mech.: Theor. Exp. P01002

[24] Feng E H and Crooks G E 2008 Phys. Rev. Lett. 101090602

[25] Horowitz J and Jarzynski C 2009 Phys. Rev. E 79021106 
[26] Parrondo J M R, Van den Broeck C and Kawai R 2009 New J. Phys. 11073008

[27] Jarzynski C 2011 Ann. Rev. Cond. Mat. Phys. 2 329-351

[28] Cover T M and Thomas J A 2006 Elements of Information Theory 2nd ed (New York: Wiley) 